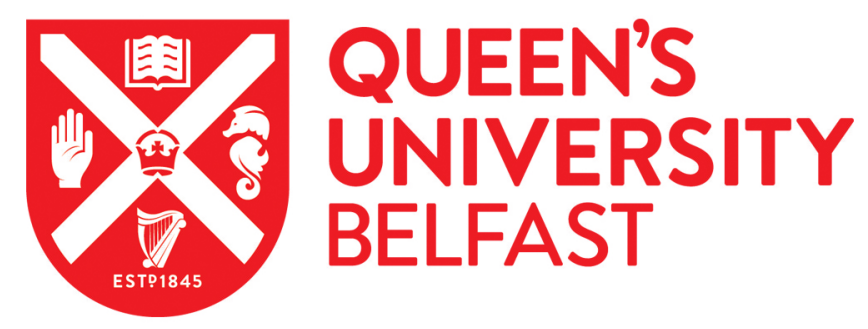

\title{
Self-Similar Nested Flux Closure Structures in a Tetragonal Ferroelectric
}

Chang, L. -W., Nagarajan, V., Scott, J. F., \& Gregg, J. M. (2013). Self-Similar Nested Flux Closure Structures in a Tetragonal Ferroelectric. Nano Letters, 13(6), 2553-2557. https://doi.org/10.1021/nl400629m

\section{Published in:}

Nano Letters

\section{Document Version:}

Peer reviewed version

Queen's University Belfast - Research Portal:

Link to publication record in Queen's University Belfast Research Portal

\author{
Publisher rights \\ (C) 2013 American Chemical Society \\ This document is the Accepted Manuscript version of a Published Work that appeared in final form in \\ Nano Letters, copyright ( American Chemical Society after peer review and technical editing by the publisher. \\ To access the final edited and published work see http://pubs.acs.org/page/policy/articlesonrequest/index.html
}

\section{General rights}

Copyright for the publications made accessible via the Queen's University Belfast Research Portal is retained by the author(s) and / or other copyright owners and it is a condition of accessing these publications that users recognise and abide by the legal requirements associated with these rights.

Take down policy

The Research Portal is Queen's institutional repository that provides access to Queen's research output. Every effort has been made to ensure that content in the Research Portal does not infringe any person's rights, or applicable UK laws. If you discover content in the Research Portal that you believe breaches copyright or violates any law, please contact openaccess@qub.ac.uk. 


\section{Self-Similar Nested Flux Closure Structures in a Tetragonal Ferroelectric}

L.-W. Chang ${ }^{1 *}$, V. Nagarajan ${ }^{2}$, J. F. Scott ${ }^{3}$ and J. M. Gregg ${ }^{1 \S}$

${ }^{1}$ Centre for Nanostructured Media, School of Maths and Physics, Queen's University Belfast, University Road, Belfast, N. Ireland, BT71NN. UK.

${ }^{2}$ School of Materials Science and Engineering, University of New South Wales, Sydney, NSW 2052, Australia

${ }^{3}$ Department of Physics, Cavendish Laboratory, J. J. Thomson Ave., Cambridge, CB3 OHE, England, UK

*email: lchang01@qub.ac.uk

§email: m.gregg@qub.ac.uk

\section{Abstract:}

In specific solid-state materials, under the right conditions, collections of magnetic dipoles are known to spontaneously form into a variety of rather complex geometrical patterns, exemplified by vortex and skyrmion structures. While theoretically, similar patterns should be expected to form from electrical dipoles, they have not been clearly observed to date: the need for continued experimental exploration is therefore clear. In this article we report the discovery of a rather complex domain arrangement that has spontaneously formed along the edges of a thin single crystal ferroelectric sheet, due to surface-related depolarizing fields. Polarization patterns are such that nanoscale 'flux-closure' loops are nested within a larger mesoscale flux closure object. Despite the orders of magnitude differences in size, the geometric forms of the dual-scale flux closure entities are rather similar. 
There is remarkable diversity and complexity in the geometric patterns that can be formed by local dipole moments in magnetic materials. Predicting and understanding these patterns has been an established topic for theoretical consideration for some time [1], but it is only relatively recently (just over a decade ago) that complex topologies, like magnetic vortices $[2,3]$, have been explicitly identified experimentally. Since initial observations, research has moved apace and now magnetic vortex objects are commonplace. In fact the vortex defines a specific domain wall type frequently found, for example, in permalloy nanowires [4,5]. Their formation, dynamics and the manner in which pinning and depinning of vortex walls can be controlled have all been studied, as part of the drive towards the development of both racetrack memory [6] and domain wall logic systems [7]. Magnetic skyrmion structures and skyrmion lattices have also been of considerable recent interest, as well as undoubted aesthetic appeal [8-10].

Although ferroelectrics are often seen as highly analogous to ferromagnets, complex electrical dipole patterns, similar to those seen in magnetics, have not yet been commonly observed. More often than not, ferroelectrics respond to the existence of depolarizing fields by forming periodic arrays of simple $180^{\circ}$ domains $[11,12]$ without the triangular flux-closure caps envisioned by Landau and Lifshitz [13]. In fact, simple flux closure arrangements of conventional $90^{\circ}$ domains, which might act as precursors indicating the possibility of genuine vortex formation (where local dipole orientation varies continuously around a geometrical core and is perpendicular to the radial vector from the core at all points), have only recently been identified [14-21]. Aberration-corrected transmission electron microscopy has revealed that continuous rotation in electrical dipoles can occur [22,23] and this is an important prerequisite for the existence of complex topological patterns. However, direct evidence for fully formed vortex states is still relatively weak [24, 25] and while skyrmion patterns have been considered in theoretical work [26], no reports have yet demonstrated electrical dipole skyrmions with anything like the weight of evidence available for magnetic equivalents. A considerable amount of research is therefore still required simply to map the kinds of topological structures that can be formed in ferroelectrics. In pushing forward this exploration, it should be noted that there are several environments in which electrical dipole pattern complexity is likely to be encouraged: in the vicinity of multiple domain wall junctions [20,21], and close to interfaces with non-compensating insulators where large depolarizing fields occur $[22,23]$.

In this article, we present the discovery of an interesting topological form in ferroelectrics, found in situations where both of the above environmental influences are in 
place. We have found a number of domain patterns at the edges of $\left[\mathrm{Pb}\left(\mathrm{Zn}_{1 / 3} \mathrm{Nb}_{2 / 3}\right) \mathrm{O}_{3}\right]_{0.88}\left[\mathrm{PbTiO}_{3}\right]_{0.12}$ (or PZN-12PT) single crystal thin film lamellae in which nanoscale flux closure loops occur near domain wall junctions and are themselves contained within a larger mesoscale flux closure object composed of bundles of $90^{\circ}$ stripe domains. Flux closure therefore occurs simultaneously at two very different length scales within the same topological entity. Intriguingly, there are geometrical similarities between these different sized flux closure objects: in both, flux closure is accommodated by stepwise rotation of polarization vectors around a loop which contains two domain wall vertices or junctions rather than a single core singularity. This repetition of the same general structure on two different scales within the same object invokes clear analogies to self-similar fractal patterns or 'Russian' nested dolls (matrioshki) and represents a new type of topology in ferroics. The thermodynamic requirement for forming a two-level flux closure object is not yet clear, but may involve both depolarizing fields and substrate-induced stress.

PZN-12PT is a tetragonal dipolar perovskite oxide, with a composition close to a morphotropic phase boundary (MPB) between a number of different symmetry states [27-29]. The proximity of a MPB was seen as advantageous in the search for complex topological patterns, as it implies that, for this composition, several symmetries are energetically similar; hence electrical dipoles might display relative freedom in their local orientation and not be overly confined into particular crystallographic orientations [30].

A FEI200TEM focused ion beam microscope (FIB) was used to cut thin lamellae $(\sim 13 \mu \mathrm{m} x \sim 12 \mu \mathrm{m} x \sim 250 \mathrm{~nm})$ of PZN-12PT with surfaces approximately parallel to $\{001\}_{\text {pseudocubic (pc) }}$ crystal planes (using the bounding sidewalls of the single crystal as reference, but also confirming orientation by transmission electron microscopy on thinner lamellae lifted out onto microporous carbon-coated copper grids). Lamellae were lifted free from their host bulk single crystal using an ex-situ micromanipulator and sharpened glass needle and placed onto $\mathrm{Si}_{3} \mathrm{~N}_{4}$ coated silicon substrates. The entire sample was then annealed in air at $600^{\circ} \mathrm{C}$ for one hour to allow FIB-induced damage of the PZN-12PT lamellar surface to be repaired and for implanted gallium to be expelled forming gallium-rich surface platelets or dots (presumably gallium oxide), using ramp rates from and to room temperature of $5^{\circ} \mathrm{Cmin}^{-1}$ and $2.5^{\circ} \mathrm{Cmin}^{-1}$ respectively. A subsequent light etching treatment with dilute $\mathrm{HCl}$ removed any gallium-rich platelets. After repeating the thermal anneal described above, a pristine surface suitable for piezoresponse force microscopy (PFM) is produced. Cooling through the Curie temperature before imaging allows domain states to react naturally to aspects of the sample geometry (such as local depolarizing fields); under these conditions 
domain arrangements closer to equilibrium are more likely than those formed under the intense and inhomogeneous externally applied fields used in prior research, where complex domain patterns were formed by fields at SPM tips $[16,18,20]$.

Subsequent domain imaging was performed using piezoresponse force microscopy (PFM) using a Veeco Dimension 3100 base microscope with a Nanoscope IIIa controller. A probing signal of $1.2 \mathrm{~V}_{\text {rms }}$ was applied to a Nanosensors PPP-EFM cantilever (force constant in the range $0.5-9.5 \mathrm{~N} / \mathrm{m}$ ), at a fixed frequency of $20 \mathrm{kHz}$. WSxM 4.0 Develop 12.6 software [31] was used for image display and processing.

Even after cursory PFM mapping, it was clear that the edges of the PZN-12PT lamellae were decorated with unusual domain patterns. Figure 1 illustrates one of a number of domain sets. Amplitude images (figure 1(a)) show sets or bundles of relatively fine scale stripe domains with domain walls which intersect the $\{001\}_{\mathrm{pc}}$ lamellar surface along $\langle 110\rangle_{\mathrm{pc}}$ directions. Given the tetragonal symmetry of the material, and that the 'vertical' cantilever deflection was observed to be dominated by buckling or flexure due to in-plane polarization components parallel to the cantilever axis (in-plane rotation of the sample by $180^{\circ}$ caused complete phase reversal of vertical PFM signal), the stripes were assigned to be $a_{1}-a_{2} 90^{\circ}$ domains with $\{110\}_{\mathrm{pc}}$ domain walls. Previous characterization of sets, or bundles, of domains has demonstrated that they can behave as collective entities, or 'superdomains' [32], in which each superdomain has a net polarization given by the vector sum of all of its constituent subdomains. This concept has been reasonably well established in literature [18, 33], as part of a wider recognition that domains are often ordered on a number of different lengthscales forming heirarchical microstructures [34-37]. The vector sum of the polarization from $a_{1}-a_{2}$ $90^{\circ}$ stripe domains of equal width lies along $\langle 110\rangle_{\mathrm{pc}}$, perpendicular to the domain walls within each superdomain, and it is instructive to interpret the overall polarization associated with the structure in figure 1 at this mesoscale level: the phase images in figure 1 (b) clearly show that the superdomain polarization in the regions on the left and right-hand side of the figure are in opposing senses; equally, in figure 1 (c), the polarizations in the top and bottom regions are also in opposing senses. Phase information (figure 1(d)) obtained with the cantilever at $45^{\circ}$ to those in figures $2(\mathrm{~b}$-c) confirms that, at a superdomain level, this object must represent an in-plane flux closure structure illustrated schematically in figure 1(e). Note that this flux closure does not contain a single 'core' intersection point of all four superdomain boundaries; rather two three-fold junctions, or 'vertices', have formed separated by a $180^{\circ}$ superdomain boundary. 
Detailed characterization of this boundary reveals further intriguing information: the area within the highlighted box in figure 1(a) has been imaged at higher resolution in two perpendicular orientations (figure $2(\mathrm{a}, \mathrm{b})$ ). By transposing the components of local polarization implied by these two orthogonal scans onto the schematic of the superdomain boundary presented in figure $2(\mathrm{c})$, the $\mathrm{a}_{1}-\mathrm{a}_{2}$ structure on either side of the boundary becomes obvious (indicated by the red arrows). Moreover, when local polarization components are considered at the vertex points along the $180^{\circ}$ superdomain wall (the central region in figure 2(c)), chains of flux-closure and quadrupole junctions are implied, similar to those written into $\mathrm{BiFeO}_{3}$ thin films by Balke et al. [20] and indirectly inferred at superdomain junctions in $\mathrm{BaTiO}_{3}$ by McGilly et al. [21]. Here, however, these nanoscale flux closure-quadrupole chains are themselves contained within a much larger meso-scale flux closure object, so that interesting polarisation topology exists simultaneously on two quite different length scales.

The schematic in figure 2(c) ignores subtle structural information, elucidated further in figure 3. Figure 3(a) presents the amplitude of the flexural signal detected with the cantilever oriented along a $<100\rangle_{\mathrm{pc}}$ direction. Needle domains are evident on both sides of the boundary. However, between the sets of needle domains, there is a distinct extended region in which the amplitude is very low. Needle domains on either side of this superdomain boundary, with polarizations parallel to the cantilever axis, are therefore distinctly separated, as illustrated by the grey shaded needle domains in the schematic of the situation given in figure 3 (d). The amplitude image in figure 3(b) shows domains with polarizations perpendicular to those in figure 3(a). Here the region of low amplitude between the domains across the $180^{\circ}$ superdomain boundary is much narrower (figure 3(c)). Domains appear to abut each other more tightly, generating more sharply delineated domain walls. When this information is transposed onto the schematic of the detailed boundary structure in figure 3 (d), it can be seen that the flux-closure loops bear a striking resemblance to those seen at the superdomain level: again domain walls do not intersect at four-fold vertices, but rather two distinct domain wall junctions form. Similarities between this local flux closure structure and that seen at a much coarser length scale in figure 1(e) are evident. We note that there is an implication of locally charged domain walls in the schematic in figure 3(d) and even though charged domain walls are progressively being observed as commonplace in ferroelectrics [38, 39], it is a surprising result of the attempted boundary reconstruction. Nevertheless, this is the form of the boundary directly implied by the PFM data. Moreover, charged states are only local as the net electrostatic flux (encapsulated by an extended Gaussian surface) from both the flux closure and quadrupole structures is necessarily zero. For reference and for clarity, 
the entire object in which both nanoscale and mesoscale flux closure is simultaneously present is illustrated schematically in figure 4.

Given that even simple flux closure objects have only recently been seen in ferroelectrics [15-21], the discovery of the nested dual-scale topologically similar flux closure states presented here establishes a new benchmark in our exploration of complexity in the spontaneous ordering of electrical dipoles. We have not demonstrated true fractal selfsimilarity, but the analogy to fractal behaviour is nevertheless obvious.

The nature of the specific geometry of the flux closure structure may be important: at both meso and nano length scales, simple quadrant flux closure states, where four $90^{\circ}$ domain boundaries intersect at a single point, are avoided. Instead, the flux closure object is split into two junction points where domain boundaries intersect. As noted in previous work [21], the reluctance of the system to form a single domain wall junction at the geometric centre or 'core' of the flux closure object could give insight into the energetics of dipole misalignment. Srolovitz and Scott [40] used two models to assess the energetic stability of different domain wall junction geometries in flux closure states: a simple Potts' model, where any degree of dipole misalignment between nearest neighbors (save for $180^{\circ}$ anti-alignment) was relatively energetically expensive, suggested that four-fold domain wall junctions were unstable with respect to the formation of two spatially separated junctions. However, by allowing some degree of dipole misalignment, through a "vector-Potts", or clock model, a four-fold flux closure core junction could be stabilised. The spatial splitting of the flux-closure objects found here suggests an intolerance to dipole misalignment at all length scales. Thus, if the Srolovitz and Scott treatment is robust, the continuous polar rotation previously seen by aberration corrected microscopy [22,23] may be relatively uncommon, even in ferroelectric compositions close to morphotropic phase boundaries (as investigated here).

The fundamental reason for the formation of the nested flux closure loops observed is not entirely obvious, but is worthy of some discussion. Of course, depolarizing fields are likely to be present at the edges of the PZN-12PT lamellae, and these should drive flux closure in some form. However, previous work on $\mathrm{BaTiO}_{3}$ [19] and $\mathrm{Pb}(\mathrm{Zr}, \mathrm{Ti}) \mathrm{O}_{3}$ [17], has already established that the effects of depolarizing fields can be successfully accommodated by flux closure at a single length scale (mesoscale). Additional internal depolarizing fields caused within a mesoscale flux closure object should not be present. Equally, the formation of flux closure-quadrupole chains is not a structural necessity - a shift in the registry of the stripe domains on either side of the $180^{\circ}$ superdomain junction by half a period would allow 
simple zig-zag $180^{\circ}$ domain walls to form instead; after all, these rather simpler superdomain boundary structures have been previously observed in $\mathrm{BaTiO}_{3}[21]$.

We note, however, that the general domain patterns in PZN-12PT were found to be strongly sensitive to the substrates onto which lamellae were mounted: when placed onto platinum-coated $\mathrm{MgO}$, domains imaged by $\mathrm{PFM}$ appeared in disordered mosaic arrangements, distinctly different from the classical tetragonal ferroelectric-ferroelastic domains evident in the figures presented herein. While such differences in domain habit have not been rationalized in detail, it seems likely that they are the result of variations in the differential thermal contraction stresses that can arise between lamella and substrate during heating and cooling cycles associated with thermal annealing. Thus one possibility is that the multiple-scale flux closure states reflect the simultaneous need to offset depolarizing fields and stress fields induced by thermal processing and differential thermal expansion between the PZN-12PT and the $\mathrm{Si}_{3} \mathrm{~N}_{4}$ coated silicon substrates used.

In summary, an interesting topological polarization object, spontaneously formed at the edge of a PZN-12PT lamella on cooling through the Curie Temperature, has been mapped using PFM. Closure loops in polarization have been observed both at the mesoscale and nanoscale, with the nanoscale closure (along with polar quadrupole structures) being spatially embedded within the mesoscale closure structure. The manner in which flux-closure has been structurally facilitated was also found to be similar in both nano and mesoscale objects, suggesting that distinct core singularities at the centre of flux-closure arrangements are energetically unfavourable. The discovery of this 'nested' topological structure extends our knowledge on the possible domain patterns that can form spontaneously in response to the significant depolarizing fields that are present in meso and nano-scale ferroelectric objects.

\section{Acknowledgements}

The authors acknowledge financial support from the Engineering and Physical Sciences Research Council (EP/H04339X/1, EP/F004869/1 and EP/F001630/1) and The Leverhulme Trust (F/00 203/V).

\section{References}

[1] Mermin, N. D. The topological theory of defects in ordered media. Rev. Mod. Phys., 1979, $51,591$.

[2] Shinjo, T.; Okuno, T.; Hassdorf, R.; Shigeto, K.; Ono, T. Magnetic vortex core observation in circular dots of permalloy. Science, 2000, 289, 930. 
[3] Wachowiak, A. et al. Direct observation of internal spin structure of magnetic vortex cores. Science, 2002, 298, 577.

[4] McMichael, R. D.; Donahue, M. J. Head to head domain wall structures in thin magnetic strips. IEEE Transactions on Magnetics, 1997, 33, 4167.

[5] Klaui, M.; Jubert, P. O.; Allenspach, R.; Bischof, A.; Bland, J. A. C.; Faini, G.; Rudiger, U.; Vaz, C. A. F.; Vila, L.; Vouille, C. Direct observation of domain-wall configurations transformed by spin currents, Phys. Rev. Lett., 2005, 95, 026601.

[6] Hayashi, M.; Thomas, L.; Moriya, R.; Rettner, C.; Parkin, S. S. P. Current-Controlled Magnetic Domain-Wall Nanowire Shift Register, Science, 2008, 320, 209.

[7] Allwood, D. A.; Xiong, G.; Faulkner, C. C.; Atkinson, D.; Petit, D.; Cowburn, R. P. Magnetic Domain Wall Logic, Science, 2005, 309, 1688.

[8] Yu, X. Z.; Onose, Y.; Kanazawa, N.; Park, J. H.; Han, J. H.; Matsui, Y.; Nagaosa, N.; Tokura, Y. Real-space observation of a two-dimensional skyrmion crystal, Nature, 2010, 465, 901.

[9] Rossler, U. K.; Bogdanov, A. N.; Pfleiderer, C. Spontaneous skyrmion ground states in magnetic metals, Nature, 2006, 442, 797.

[10] Muhlbauer, S.; Binz, B.; Jonietz, F.; Pfleiderer, C.; Rosch, A.; Neubauer, A.; Georgii, R.; Boni, P. Skyrmion Lattice in a Chiral Magnet, Science, 2009, 323, 915.

[11] Mitsui, T.; Furuichi, J. Domain Structure of Rochelle Salt and $\mathrm{KH}_{2} \mathrm{PO}_{4}$, Phys. Rev., 1953, 90, 193.

[12] Speck, J. S.; Pompe, W., Domain configurations due to multiple misfit relaxation mechanisms in epitaxial ferroelectric thin films. I. Theory, J. Appl. Phys., 1994, 76, 466.

[13] Landau, L. D.; Lifshitz, E. M. On the theory of the dispersion of magnetic permeability in ferromagnetic bodies, Phys. Z. Sowjetunion, 1935, 8, 153.

[14] Slutsker, J.; Artemev, A.; Roytburd, A. Phase Field Modeling of Domain Structure of Confined Nanoferroelectrics, Phys. Rev. Lett., 2008, 100, 087602.

[15] Tsou, N. T.; Potnis, P. R.; Huber, J. E. Classification of laminate domain patterns in ferroelectrics, Phys. Rev. B, 2011, 83, 184120.

[16] Vasudevan, R. K.; Chen, Y.-C.; Tai, H.-H.; Balke, N.; Wu, P.; Bhattacharya, S.; Chen, L. Q.; Chu, Y.-H.; Lin, I.-N.; Kalinin, S. V.; Nagarajan, V. Exploring Topological Defects in Epitaxial BiFeO3 Thin Films. ACS Nano, 2011, 5, 879.

[17] McGilly, L. J.; Gregg, J. M. Polarisation Closure in $\operatorname{PbZr}_{(0.42)} \operatorname{Ti}_{(0.58)} \mathrm{O}_{3}$ Nanodots, Nano Lett., 2011, 11, 4490. 
[18] Ivry, Y.; Chu, D. P.; Scott, J. F.; Durkan, C. Flux Closure Vortexlike Domain Structures in Ferroelectric Thin Films. Phys. Rev. Lett., 2010, 104, 207602.

[19] McQuaid, R. G. P.; McGilly, L. J.; Sharma, P.; Gruverman, A.; Gregg, J. M. Mesoscale flux-closure domain formation in single-crystal $\mathrm{BaTiO}_{3}$. Nat. Commun., 2011, 2, 404.

[20] Balke, N.; Choudhury, S.; Jesse, S.; Huijben, M.; Chu, Y. H.; Baddorf, A. P.; Chen, L. Q.; Ramesh, R.; Kalinin, S. V. Deterministic control of ferroelastic switching in multiferroic materials. Nat. Nanotechnol., 2009, 4, 868.

[21] McGilly, L. J.; Schilling, A.; Gregg, J. M. Domain Bundle Boundaries in Single Crystal $\mathrm{BaTiO}_{3}$ Lamellae: Searching for Naturally Forming Dipole Flux-Closure/Quadrupole Chains. Nano Lett., 2010, 10, 4200.

[22] Jia, C.-L.; Urban, K. W.; Alexe, M.; Hesse, D.; Vrejoiu, I. Direct Observation of Continuous Electric Dipole Rotation in Flux-Closure Domains in Ferroelectric $\mathrm{Pb}(\mathrm{Zr}, \mathrm{Ti}) \mathrm{O}_{3}$. Science, 2011, 331, 1420.

[23] Nelson, C. T.; Winchester, B.; Zhang, Yi; Kim, S.-J.; Melville, A.; Adamo, C.; Folkman, C. M.; Baek, S.-H.; Eom, C.-B.; Schlom, D. G.; Chen, L.-Q.; Pan, X. Spontaneous Vortex Nanodomain Arrays at Ferroelectric Heterointerfaces. Nano Lett., 2011, 11, 828.

[24] Gruverman, A.; Wu, D.; Fan, H.-J.; Vrejoiu, I.; Alexe, M.; Harrison, R. J.; Scott, J. F. Vortex ferroelectric domains. J. Phys.: Cond. Matt., 2008, 20, 342201.

[25] Rodriguez, B. J.; Gao, X. S.; Liu, L. F.; Lee, W.; Naumov, I. I.; Bratkovsky, A. M.; Hesse, D.; Alexe, M. Vortex polarization states in nanoscale ferroelectric arrays. Nano Lett., 2009, 9, 1127.

[26] Baudry, Laurent; Sene, Anais; Luk'yanchuk, Igor A.; Lahoche, Laurent. Vortex state in thin films of multicomponent ferroelectrics, Thin Solid Films, 2011, 519, 5808.

[27] Noheda, B.; Cox, D. E.; Shirane, G.; Park, S. E.; Cross, L. E.; Zhong, Z. Polarization rotation via a monoclinic phase in the piezoelectric $92 \% \mathrm{~Pb}\left(\mathrm{Zn}_{1 / 3} \mathrm{Nb}_{2 / 3}\right) \mathrm{O}_{3}-8 \% \mathrm{PbTiO}_{3}$ Phys. Rev. Lett., 2001, 86, 3891.

[28] Cox, D. E.; Noheda, B.; Shirane, G.; Uesu, Y.; Fujishiro, K.; Yamada, Y. Universal phase diagram for high-piezoelectric perovskite systems, Appl. Phys. Lett. 2001, 79, 400.

[29] Noheda, B. Structure and high-piezoelectricity in lead oxide solid solutions, Current Opinion in Solid State \& Materials Science 2002, 6, 27.

[30] Fu, H. X.; Cohen, R. E. Polarization rotation mechanism for ultrahigh electromechanical response in single-crystal piezoelectrics, Nature 2000, 403, 281.

[31] Horcas, I.; Fernandez, R.; Gomez-Rodriguez, J. M.; Colchero, J.; Gomez-Herrero J.; Baro, A. M., WSXM: A software for scanning probe microscopy and a tool for nanotechnology, Rev. Sci. Instrum. 2007, 78, 013705. 
[32] McGilly, L. J.; Gregg, J. M. Scaling of Superdomain Bands in Ferroelectric Dots, Appl. Phys. Lett., 2011, 98, 132902.

[33] Streiffer, S. K.; Parker, C. B.; Romanova, A. E.; Lefevre, M. J.; Zhao, L.; Speck, J. S; Pompe, W.; Foster, C. M.; Bai, G. R. Domain patterns in epitaxial rhombohedral ferroelectric films.I. Geometry and experiments, J. Appl. Phys., 1998, 83, 2742.

[34] Roitburd, A. L.; Equilibrium structure of epitaxial layers, Phys. Status Solidi A, 1976, 37, 329.

[35] Arlt, G.; Sasko, P. Domain configuration and equilibrium size of domains in $\mathrm{BaTiO}_{3}$ ceramics, J. Appl. Phys., 1980, 51, 4956.

[36] Hooton J.; Merz, W. Etch Patterns and Ferroelectric Domains in $\mathrm{BaTiO}_{3}$ Single Crystals, Phys. Rev., 1955, 98, 409.

[37] Tsou, N. T.; Huber, J. E.; Cocks, A. C. F. Evolution of compatible laminate domain structures in ferroelectric single crystals, Acta Materialia, 2013, 61, 670.

[38] Sluka, T.; Tagantsev, A.K.; Damjanovic, D.; Gureev, M.; Setter, N. Enhanced electromechanical response of ferroelectrics due to charged domain walls. Nature Communications, 2012, 3, 748.

[39] Meier, D. et al. Anisotropic conductance at improper ferroelectric domain walls. Nature Materials, 2012, 11, 284.

[40] Srolovitz, D. J.; Scott, J. F. Clock-model description of incommensurate ferroelectric film and of nematic-liquid-crystal films. Phys. Rev. B, 1986, 34, 1815. 


\section{Figure Captions}

Figure 1: Domain structures at the edges of the PZNPT single crystal lamella were found to consist of blocks or bundles of $\mathrm{a}_{1}-\mathrm{a}_{2} 90^{\circ}$ stripe domains, visible in both amplitude (a) and phase images (b-d). When the resultant polarization vector associated with each bundle is determined (acknowledging that the image in (c) is dominantly the result of flexural cantilever distortion due to in-plane polarization components parallel to the cantilever axis), the flux closure object shown schematically in (e) is implied. The dimensions associated with this flux-closure object are of the order of microns.

Figure 2: Detailed lateral-mode PFM imaging, of the region marked by the box superposed onto the PFM amplitude image in figure 1(a), reveals distinctive phase contrast $(a, b)$. Selfconsistent analysis of this phase information allows the polarization states, on either side of the boundary between two bundles of $\mathrm{a}_{1}-\mathrm{a}_{2}$ domains, to be unequivocally determined. At the nanoscale, polarization orientations imply that the boundary consists of chains of flux closure and quadrupole structures.

Figure 3: Further detailed PFM imaging shows that stripe domains with polarization parallel (or antiparallel) to the cantilever axis form into needle-shapes that fall short of the superdomain boundary (a). These domains are represented by the grey coloured needles in the schematic reconstruction of the boundary geometry in (d). Stripe domains with polarization perpendicular to the cantilever axis, imaged through LPFM in (b), maintain piezoelectric amplitude signal almost until the domains abut each other at the superdomain wall. The inferred forms of these domains are represented by the white paddle-headed stripes in the schematic reconstruction of the boundary (d). In (c), two sections of the amplitude signal taken from (a) and (b) are compared, illustrating the difference in the relative separation of needle domains with different polarization directions. The inferred flux closure loops contained within the shaded purple parallelograms in (d) do not contain a single core point where all domain walls intersect. While not identical, there are clear similarities between the flux closure structures imaged at the nanoscale and at the mesoscale (figure 1) in this topologically complex domain configuration.

Figure 4: A summary schematic is presented to convey how the micron-scale flux closure at the superdomain level (presented explicitly in figure 1) and the nanoscale flux closure loops 
and quadrupole junctions (presented explicitly in figure 3) are integrated within a single domain pattern.
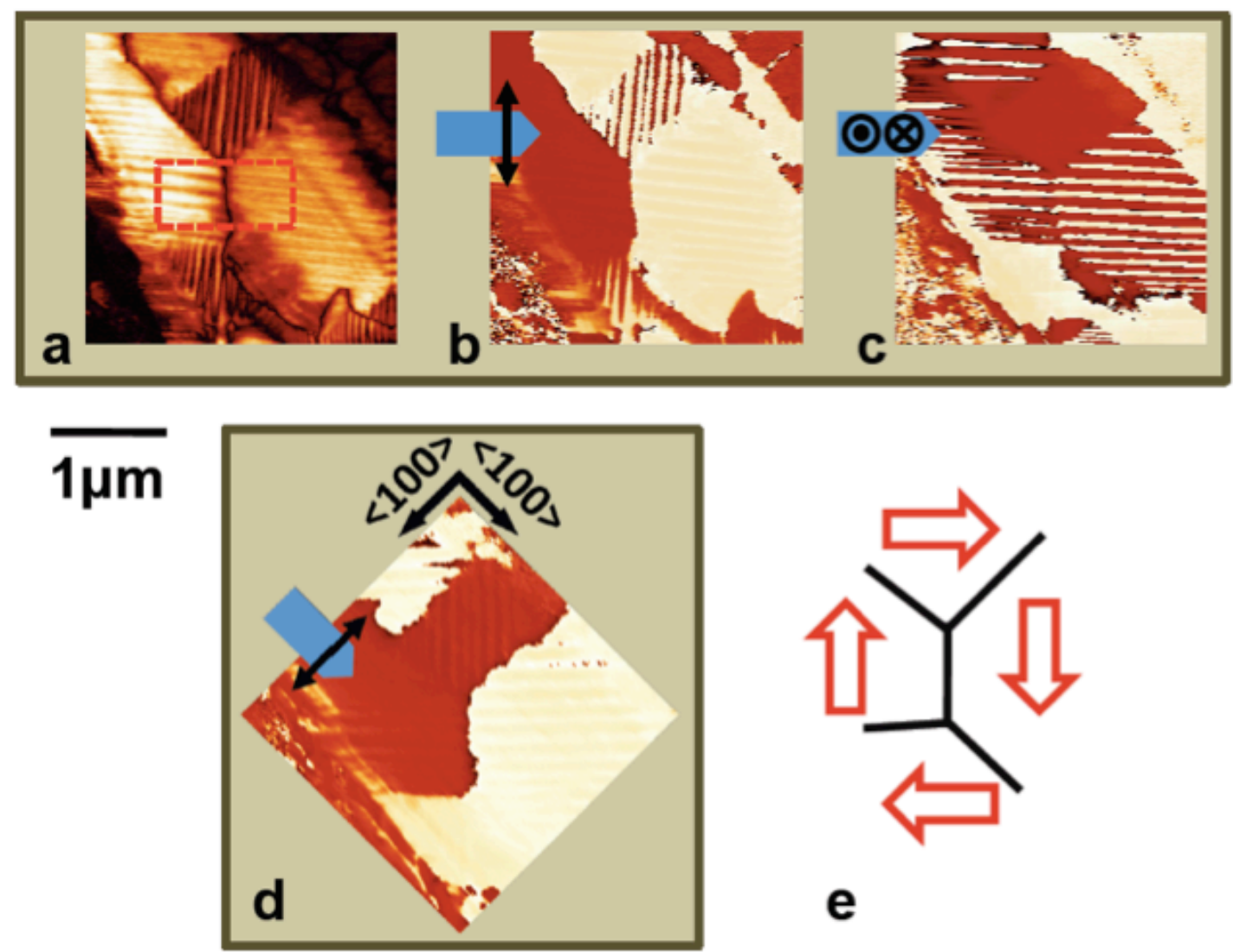

Figure 1 of 4 


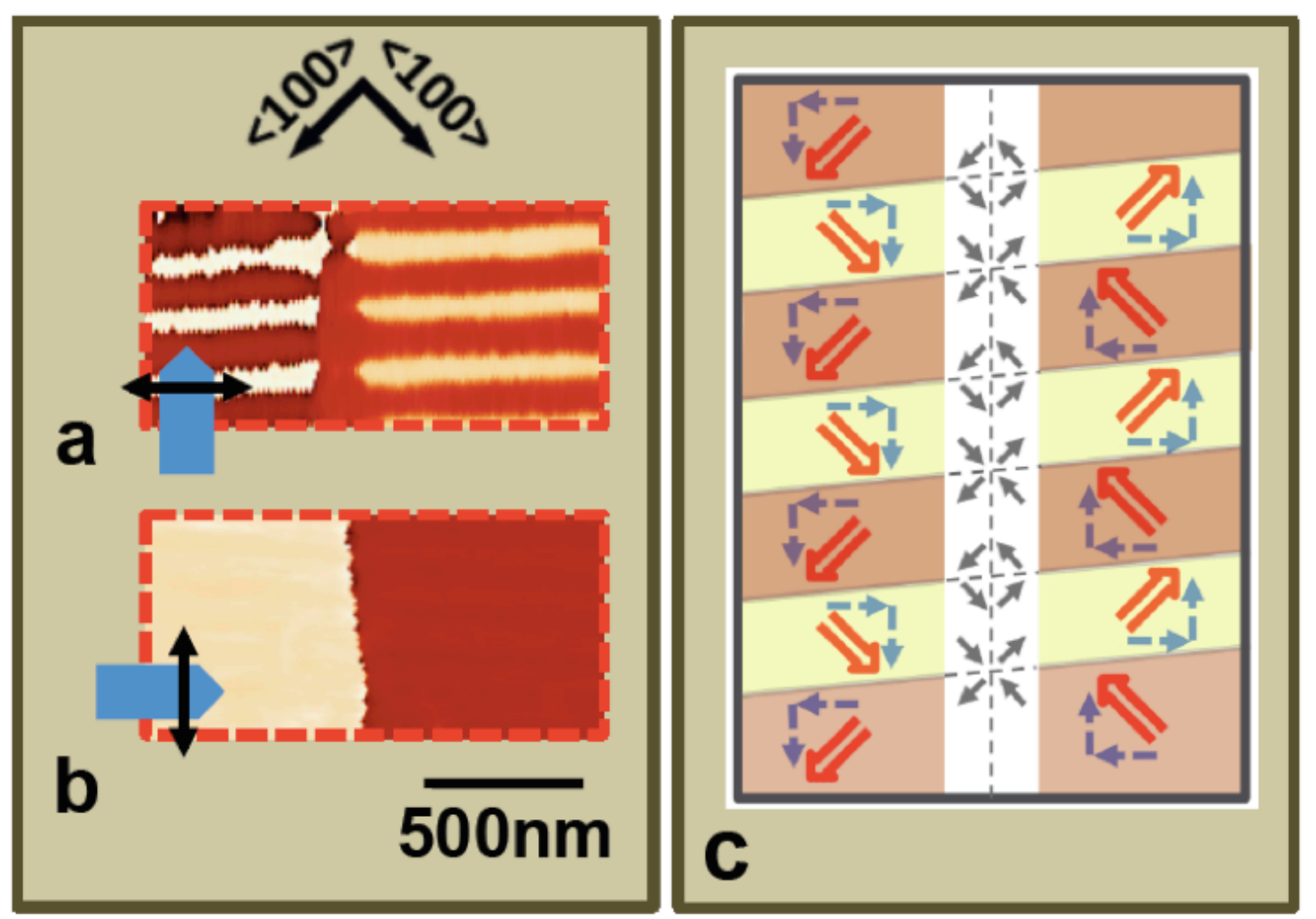

Figure 2 of 4 

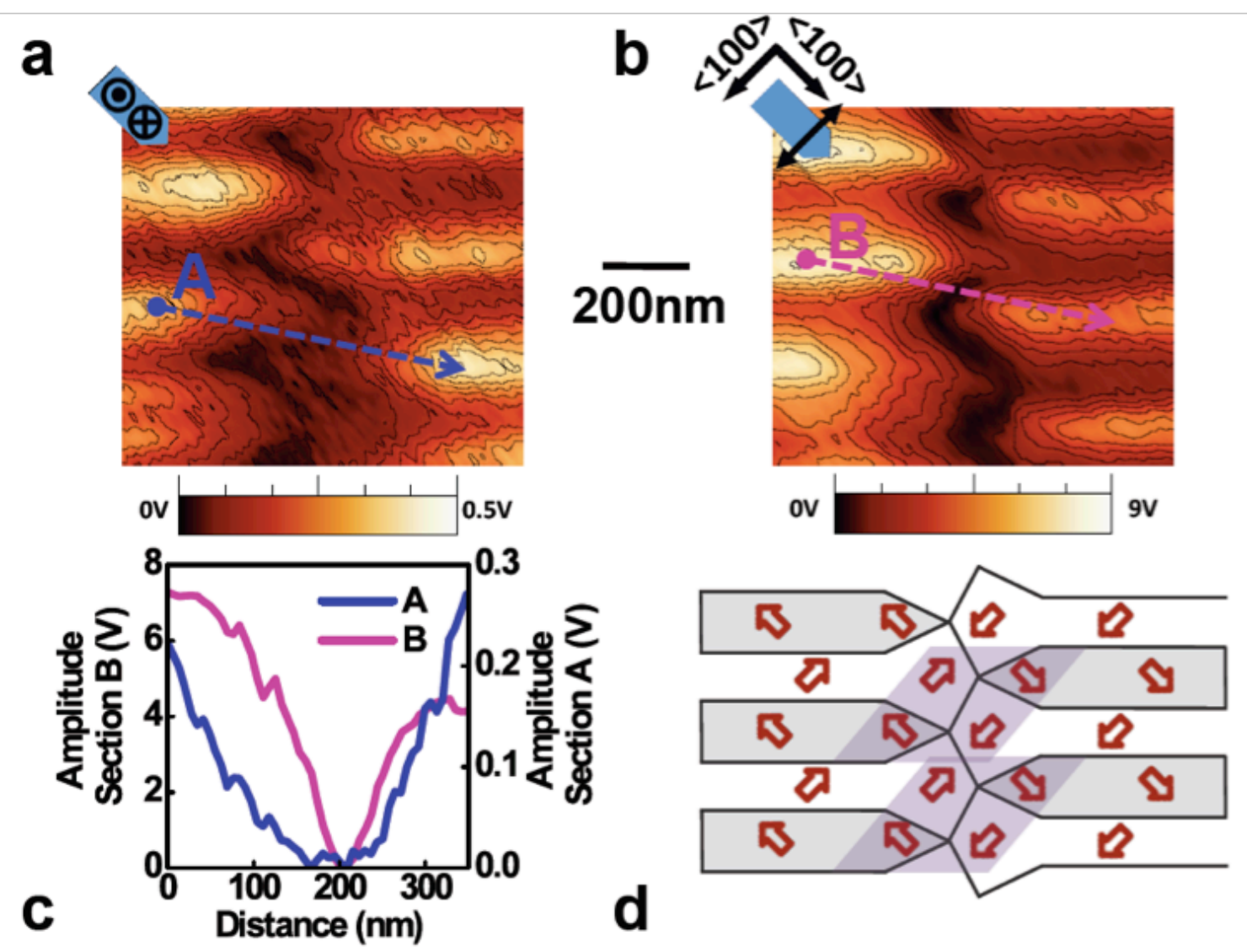

Figure 3 of 4 


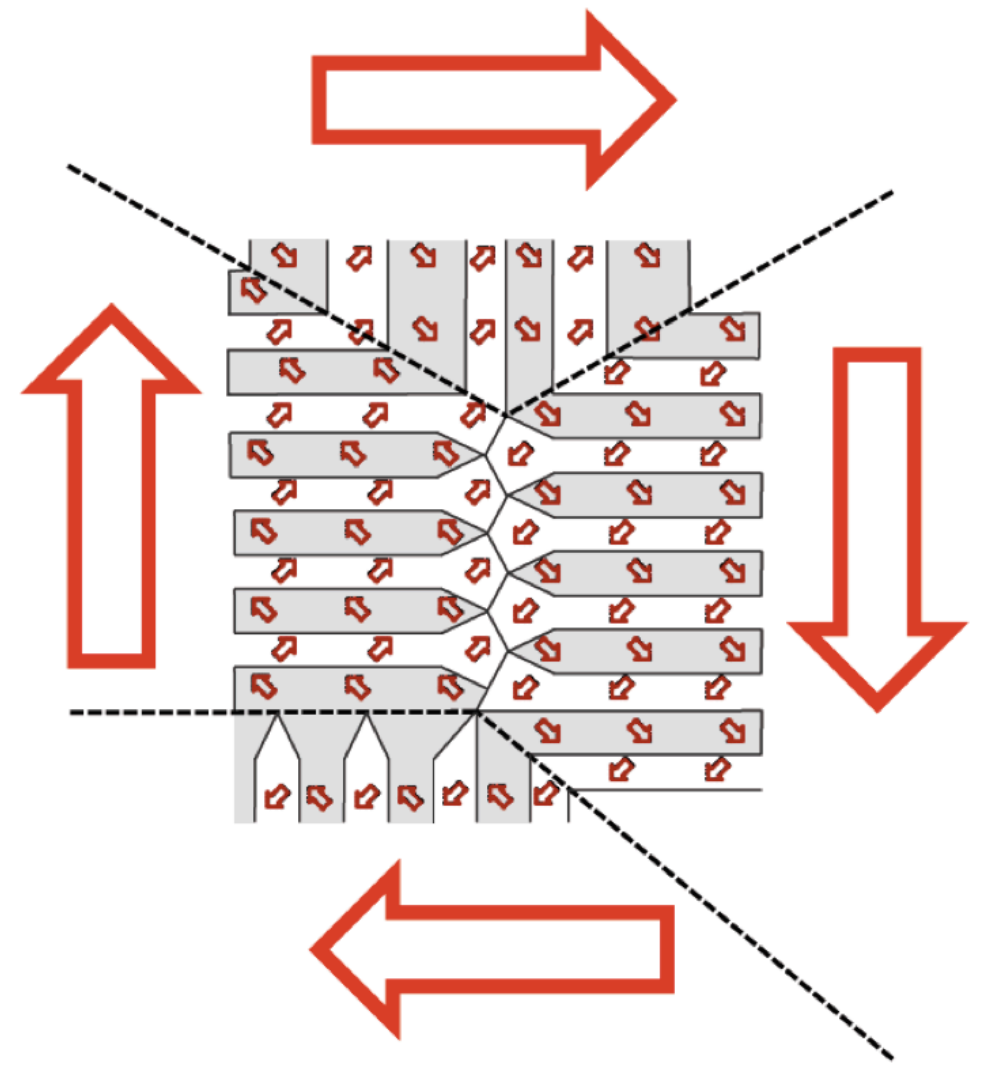

Figure 4 of 4 